\section{Occurrence of Rheumatoid Arthritis in a Patient Treated with Anakinra for Schnitzler Syndrome: A Case Report}

\section{To the Editor:}

We report a 65-year-old patient with Schnitzler syndrome evolving over 18 years who developed rheumatoid arthritis (RA) while being successfully treated for 8 years with the interleukin 1 (IL-1) inhibitor anakinra. This observation raises the question of the potential link between persistent IL-1 inhibition and RA.

A 65-year-old man had typical Schnitzler syndrome diagnosed 16 years ago on account of a 2-year history of urticarial rash, intermittent fever of up to $40^{\circ} \mathrm{C}$, pain in both tibias, and a monoclonal immunoglobulin $\mathrm{M}(\operatorname{IgM}) \kappa$ component. Prednisone, ibuprofen, and interferon- $\alpha$ proved ineffective to control his symptoms. In February 2007, treatment with anakinra (100 $\mathrm{mg} /$ day) was started. Efficiency was immediate and spectacular. In January 2015 , the patient, who was still receiving anakinra daily, complained of new symptoms: inflammatory arthralgia of the hands, wrists, and knees. He had no fever, no urticarial skin rash, and no extraarticular pain. In addition, he did not have suggestive signs of Waldenström macroglobulinemia, such as constitutional symptoms, signs indicative of hyperviscosity, or anemia. On examination, swelling and tenderness of both wrists and of all metacarpophalangeal joints and proximal interphalangeal joints were observed. Laboratory investigations showed a C-reactive protein lower than $4 \mathrm{mg} / 1$, a normal leukocytes count, and an increased erythrocyte sedimentation rate of $106 \mathrm{~mm} / \mathrm{h}$, probably related to the monoclonal IgM spike of $27 \mathrm{~g} / \mathrm{l}$, stable for about 5 years. Rheumatoid factor increased to 152 IU/l (ELISA), IgG antibodies against citrullinated peptides were highly positive at $1600 \mathrm{U} / 1$, and antinuclear antibodies were detected at 1/320 without specificity. Bone scintigraphy showed inflammatory damage of metacarpophalangeal and proximal interphalangeal joints and wrists. Joint radiographs of the hands, wrists, knees, and feet were normal. A diagnosis of RA was established and treatment with methotrexate (MTX; $15 \mathrm{mg} /$ week) was started. Four months after the beginning of MTX (raised at $20 \mathrm{mg} / w e e k$ ), the pain and swelling had entirely regressed. Anakinra was not interrupted because as soon as the patient stopped taking anakinra, symptoms of Schnitzler syndrome (high fever, rash, bone pain) recurred within $48 \mathrm{~h}$.

Our observation raises the question of whether the occurrence of RA in this patient with Schnitzler syndrome treated by anakinra is a mere coincidence or causally related. Schnitzler syndrome is a paradigm of an
IL-1-mediated acquired autoinflammatory syndrome and patients have a complete and sustained response to anakinra ${ }^{1,2}$. About 300 cases of this rare syndrome have been reported ${ }^{3}$, but not a single patient has so far developed RA, making the relationship between these disorders unlikely. Of note, although it is approved in RA, anakinra is relatively rarely prescribed in this condition because of its modest therapeutic effect. The main question is whether sustained and longterm IL-1 blockade can contribute to the pathogenesis of RA, as 1 type of paradoxal effect, by analogy to the psoriasiform rashes observed in patients treated with tumor necrosis factor inhibitors. It is, of course, impossible to answer this question on account of this single case, and the occurrence of RA in this patient treated by anakinra might be just a coincidence. Only the longterm followup of patients treated continuously with IL-1 inhibitors, such as patients with cryopyrin-associated periodic syndromes or Schnitzler syndrome, will provide a definite answer. Further, our observation underlines the complete response of Schnitzler syndrome to anakinra, so that any symptom under treatment must be evaluated to not be wrongly attributed to Schnitzler syndrome.

FANNY MARGERIN, Intern, Faculté de Médecine, Université de Strasbourg, Department of Dermatology, Hôpitaux Universitaires; JACQUES ERIC GOTTENBERG, MD, PhD, Faculté de Médecine, Université de Strasbourg, Department of Rheumatology, Hôpitaux Universitaires; DAN LIPSKER, MD, PhD, Faculté de Médecine, Université de Strasbourg, Department of Dermatology, Hôpitaux Universitaires, Strasbourg, France. Address correspondence to Dr. D. Lipsker, 1 place de l'hôpital, F-67091 Strasbourg Cedex, France.

E-mail:dan.lipsker@chru-strasbourg.fr

\section{REFERENCES}

1. Lipsker D. The Schnitzler syndrome. Orphanet J Rare Dis 2010;5:38.

2. Néel A, Henry B, Barbarot S, Masseau A, Perrin F, Bernier C, et al. Long-term effectiveness and safety of interleukin-1 receptor antagonist (anakinra) in Schnitzler's syndrome: a French multicenter study. Autoimmun Rev 2014;13:1035-41.

3. de Koning HD. Schnitzler's syndrome: lessons from 281 cases. Clin Transl Allergy 2014;5:4-41.

J Rheumatol 2016;43:7; doi:10.3899/jrheum.151466 